\title{
Research on a novel passive islanding detection method
}

In distributed generation systems, islanding detection is a necessary function of grid-connected inverters. In view of the performance disadvantages of traditional passive and active islanding detection methods, this paper proposes a novel passive islanding detection method. The proposed method first extracts characteristic parameters from the inverter output voltage signal and inverter output current signal through lifting wavelet transform, and then conducts the pattern recognition of these extracted characteristic parameters via BP neural network, so as to judge if there is an islanding phenomenon. As verified by the simulation and experiment results, the islanding detection method proposed in this paper is effective, and is featured by high detection speed and small non-detection zone, without affecting electric energy quality; its detection performance has been remarkably improved in comparison with that of traditional islanding detection methods.

Keywords: Inverter, islanding detection, lifting wavelet, neural network.

\subsection{Introduction}

$\mathrm{T}$ The renewable energy based distributed generation (DG) technology has seen a rapid development all over the world in recent years. In DG systems, renewable energies are first transformed into electric energy and then transmitted to the grid through grid-connected inverters, which poses challenges to the safe and stable operation of the grid, a major one of which is the islanding detection of grid-connected inverters. The so-called "islanding" refers to the phenomenon in which, due to the interruption of power supply by the grid as a result of failure or power-cut maintenance, the inverter still transmits electric energy to the grid and consequently forms a self-contained power supply island with local load which cannot be controlled by the public grid system. When a DG system is in the islanding operation state, it will endanger the safety of maintenance personnel, and if the islanding phenomenon is not eliminated before the grid is reclosed, it will result in asynchronous grid

Messrs. Dong Xie, Dajin Zang, Peng Gao, Junjia Wang and Zhu Zhu, Department of Electrical Engineering, Tongling University, Tongling, 244000, China. E-mail: XDY@tlu.edu.cn connection and may damage electric equipment. Thus, it is of great importance to quickly and accurately detect the islanding phenomenon of DG system and adopt related antiislanding protection measures $[1,2,3,4]$.

Currently, the inverter-based islanding detection methods can be divided into the following two types: passive detection methods and active detection methods. Passive islanding detection methods are based on the fact that the islanding phenomenon is generally accompanied by the remarkable changes of voltage, frequency, phase and so forth, and we can judge the occurrence of islanding through detecting these electric parameters to see if their changes have exceeded the set thresholds. Common passive detection methods include the overvoltage/under voltage and over frequency/under frequency detection method, the harmonics detection method, the phase jump detection method and the detection method of key electric parameters change rate. Active islanding detection methods are conducted through injecting a disturbance signal into the voltage or current control signal of the inverter, as the disturbance signal causes the amplitude or frequency of inverter output voltage to continuously deviate from normal and exceed the threshold after the occurrence of islanding, thus detecting the phenomenon of islanding. Common active detection methods include the active frequency drift method, the Sandia frequency shift method, the slip-mode frequency shift method, the impedance measurement method and the output power disturbance method $[5,6,7,8]$.

Passive islanding detection methods exert no influence on the quality of power supply, but they have the disadvantages of long detection time and large non-detection zone (NDZ), and easily results in wrong detection and missed detection. Active islanding detection methods have overcome the disadvantages of passive islanding detection methods and are featured by relatively high detection speed and remarkably reduced NDZ, but, due to the introduction of the disturbance signal, they have increased the harmonics in the inverter output current and reduced the quality of the electric energy provided by the DG system.

Aimed at the disadvantages of traditional passive and active islanding detection methods, this paper proposes a 
novel passive islanding detection method based on lifting wavelet and neural network. This method first extracts characteristic parameters from related signals of the gridconnected inverter through lifting wavelet transform, and then conducts the pattern recognition of these extracted characteristic parameters via BP neural network, so as to judge if there is an islanding phenomenon. As indicated by the simulation and experiment results, the proposed islanding detection method is featured by extremely high accuracy and reliability, has not only overcome the disadvantages of long detection time and large NDZ of traditional passive islanding detection methods, but also avoided the adverse influence of active islanding detection methods on the quality of power supply $[9,10]$.

\subsection{Passive islanding detection technology based on lifting wavelet and neural network}

\subsection{OVERVIEW OF ISLANDING DETECTION PRINCIPLE}

The principle of the proposed islanding detection method is shown in Fig.1. First, collect inverter output voltage value and inverter output current value, subject of the collected voltage and current values to lifting wavelet transform to obtain the related wavelet coefficients; then, provide algorithm processing for the obtained wavelet coefficients; finally, adopt the processed signal as the characteristic vector space of BP neural network, provide it to the input layer neurons of neural network, and use the neural network to judge the working state of the DG system according to the input characteristic vector: if the system is detected to be in the islanding state, anti-islanding protection will be provided; if the system is detected to be in the grid-connected state, no anti-islanding protection will be provided.

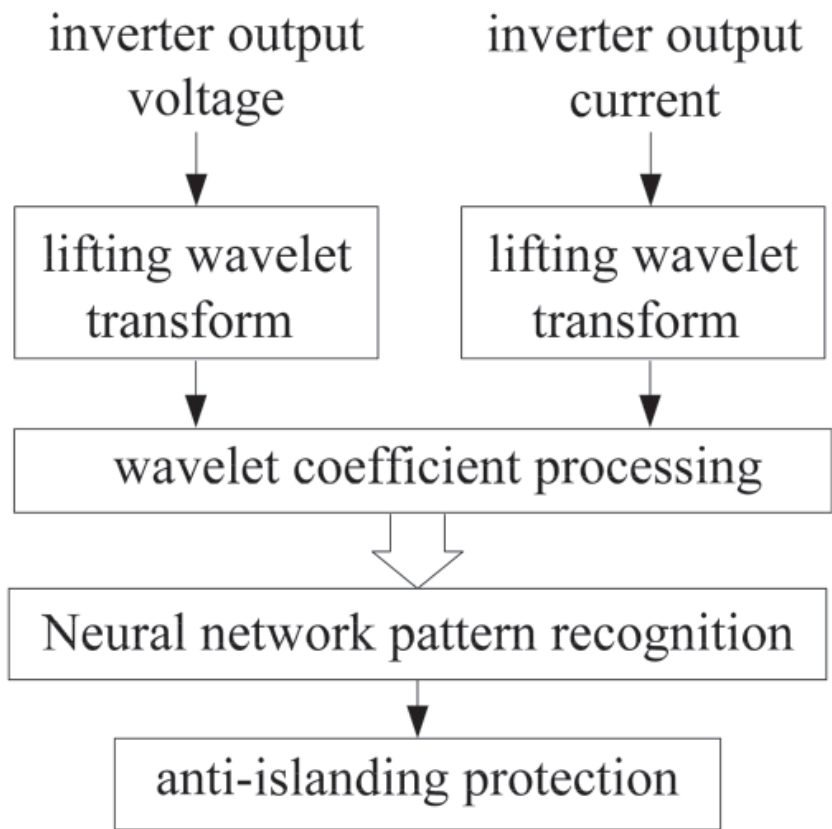

Fig.1: Principle of islanding detection
2.2 LIFTING WAVELET TRANSFORM AND SIGNAL CHARACTERISTIC PARAMETER EXTRACTION

\subsubsection{Principle of lifting wavelet transforms}

Wavelet transform is the most effective method for analyzing non-stationary signals or signals with singularity mutations, and applies to the occasions where transient mutation signals are generated, such as islanding detection. Compared to traditional wavelet algorithms which impose a heavy calculation burden and consume a lot of time, the lifting wavelet algorithm is featured by simple algorithm and quick calculation, and can complete wavelet transform at the current position without having to allocate additional memory, thus facilitating the realization of chips. Lifting wavelet transform does not depend on Fourier transform, and can satisfactorily overcome the boundary problem of wavelet transform; based on the Euclidean algorithm, all the traditional wavelets can be constructed through the lifting scheme. In view of the above advantages of lifting wavelet, this paper adopts lifting wavelet transform to extract the signal characteristic parameters needed by islanding detection [11].

The basic process of lifting wavelet transform can be divided into three links: split, prediction and update, the latter two of which are introduced to realize the high and low frequency separation of signals.

\section{(1) Split}

Split means to split an original signal $s_{j}=\left\{s_{j, k}\right\}$ into two mutually disjoint subsets, the length of each subset is half of that of the original set. Usually, a sequence is split into the even sequence $e_{j-1}$ and the odd sequence $o_{j-1}$, that is,

$\operatorname{Split}\left(s_{j}\right)=\left(e_{j-1}, o_{j-1}\right)$

Where $e_{j-1}=\left\{e_{j-1, \mathrm{k}}=s_{j, 2 \mathrm{k}}\right\}, o_{j-1}=\left\{o_{j-1, k}=s_{j, 2 \mathrm{k}+1}\right\}$. Such decomposition is referred to as inert wavelet transform.

\section{(2) Prediction}

Given the certain correlation between the even sequence and the odd sequence, the even sequence $e_{j-1}$ can be used to predict the odd sequence $o_{j-1}$. The difference $d_{j-1}$ between the actual value $o_{j-1}$ and the predicted value $P\left(e_{j-1}\right)$ reflects the degree of approximation between the two, which is referred to as wavelet coefficient and corresponds to the highfrequency component of the original signal $s_{j}$. If the prediction is rational, the information contained in the difference dataset $d_{j-1}$ will be much less than the information contained in the original set $o_{j-1}$. The prediction process can be expressed as:

$$
d_{j-1}=o_{j-1}-P\left(e_{j-1}\right)
$$

Where the prediction operator $P$ is expressed by the prediction function $P_{k}$, which can be set at the corresponding data in $e_{j-1}$.

$$
P_{k}\left(e_{j-1}\right)=e_{j-1, \mathrm{k}}=s_{j, 2 \mathrm{k}}
$$

Or it can be set at the mean value of the corresponding data in $\mathrm{e}_{\mathrm{j}-1}$ and its adjacent data: 
$\mathrm{P}_{\mathrm{k}}\left(\mathrm{e}_{\mathrm{j}-1}\right)=\left(\mathrm{e}_{\mathrm{j}-1, \mathrm{k}}+\mathrm{e}_{\mathrm{j}-1, \mathrm{k}+1}\right) / 2=\left(\mathrm{s}_{\mathrm{j}, 2 \mathrm{k}}+\mathrm{s}_{\mathrm{j}, 2 \mathrm{k}+2}\right) / 2$

$P_{k}$ can also be expressed by a more complex function.

(3) Update

The prediction process may witness the loss of some signal characteristics which happen to be the expected useful information, such as the mean value of the signal. To recover these characteristics lost in the prediction process, the update operator $\mathrm{U}$ is introduced in the following update process:

$$
s_{j-1}=e_{j-1}+U\left(d_{j-1}\right)
$$

Where $s_{j-1}$ is the low-frequency component of $s_{j}$; just like the prediction function, the update operator can also be expressed by different functions, such as

$$
U_{k}\left(d_{j-1}\right)=d_{j-1, \mathrm{k} / 2}
$$

Or

$$
U_{k}\left(d_{j-1}\right)=\left(d_{j-1, \mathrm{k}-1}+d_{j-1, k}\right) / 4+1 / 2
$$

Expressing $P$ and $U$ with different functions constructs different wavelet transforms.

Through wavelet lifting, the signal $s_{j}$ can be decomposed into the low-frequency component $s_{j-1}$ and high-frequency component $d_{j-1}$; the low-frequency data subset $s_{j-1}$ can also be subject to split, prediction and update in the same manner and be further decomposed into $s_{j-2}$ and $d_{j-2}$. In this way, after $n$ times of decomposition, the wavelet coefficients of the original data $s_{j}$ can be expressed as $\left\{s_{j-n}, d_{j-n}, d_{j-n+1}, \ldots, d_{j-1}\right\}$, wherein, $s_{j-n}$ represents the low-frequency component of the signal and $\left\{d_{j-n}, d_{j-n+1}, \ldots, d_{j-1}\right\}$ represent the high-frequency component coefficients of the signal from low to high. The decomposition corresponds to the above three lifting steps: split, prediction and update.

\subsubsection{Extraction and processing of signal characteristic parameters}

When lifting wavelet is used to decompose a signal, the decomposition will cause the lengths of the low-frequency and high-frequency components of the signal to be cut by half, and, with the increase of the decomposition scale, the numbers of data points of low-frequency and high-frequency components will become less and less. Redundant lifting wavelet transform adopts interpolation zero-padding operation for the predictors and updaters of different layers, thus ensuring that the lengths of low-frequency and highfrequency components are equal to that of the original signal and providing richer characteristic data. On that account, this paper selects redundant lifting wavelet transform to extract the characteristic parameters of related signals, with the purpose of making a sound judgment about islanding state and non-islanding state.

According to the real-time requirements on classification accuracy, detection speed and hardware service, the signal sampling frequency for islanding detection was set at $10 \mathrm{KHz}$ in this paper; according to Shannon's Theorem, there can be a maximum of seven layers of decomposition above the fundamental frequency, and the high-frequency components of the seven layers are represented by $D_{1} \sim D_{7}$, respectively; see the decomposition levels and frequency band range in Table 1.

TABLE 1: WAVELET DECOMPOSITION LEVELS AND FREQUENCY BAND

\begin{tabular}{ccc}
\hline $\begin{array}{c}\text { Decomposition } \\
\text { levels }\end{array}$ & $\begin{array}{c}\text { High-frequency } \\
\text { components }\end{array}$ & $\begin{array}{c}\text { Frequency } \\
\text { bandrange }\end{array}$ \\
\hline 1 & D1 & $2500 \sim 5000$ \\
2 & D2 & $1250 \sim 2500$ \\
3 & D3 & $625 \sim 1250$ \\
4 & D4 & $312.5 \sim 625$ \\
5 & D5 & $156.25 \sim 312.5$ \\
6 & D6 & $78.125 \sim 156.25$ \\
7 & D7 & $39.0625 \sim 78.125$ \\
\hline
\end{tabular}

Before using lifting wavelet and neural network technology for islanding detection, it is necessary to select some voltage or current signals from the DG system to construct characteristic parameters. However, when extracting voltage signal characteristic parameters or current signal characteristic parameters alone for islanding identification, the characteristic parameters extracted at islanding state and those extracted at non-islanding state will be very approximate under certain loading conditions, which will further result in mode mixing and causes the neural network to make a misjudgment in identifying the islanding mode. For that reason, this paper simultaneously extracts the characteristic parameters of both inverter output voltage and inverter output current, for the purpose of identifying the islanding mode.

The general processing method of extracting signal characteristic parameters through wavelet transform is to solve the norms of all the wavelet coefficients of the highfrequency component of each layer. To reduce the input signal value of neural network and prevent an excessively large absolute value of its net input from causing the nerve cell output to be saturated and further affecting the learning convergence speed of neural network, instead of calculating the norm of the wavelet coefficient, this paper chooses to calculate the absolute mean value of the wavelet coefficient as the input signal of neural network. In addition, to reduce the degree of complexity of neural network and to lighten the calculation burden for the purpose of improving the real-time performance of islanding detection, the number of characteristic parameters input into the neural network should be controlled. On that account, under the premise of guaranteeing the accuracy rate of identification, the characteristics significantly affecting the identification results can be retained, while those having an insignificant influence can be stripped out. By comparing the absolute mean values of the wavelet coefficients of seven scales obtained at both islanding state and non-islanding state, the absolute mean 
values of the wavelet coefficients of layer $\mathrm{D}_{1}$ and layer $\mathrm{D}_{2}$ were selected for the mode identification of neural network. To enlarge the differences among characteristic vectors of the system at different working states and ensure that the neural network can make an accurate judgment about the islanding state, six signals, consisting of the absolute mean values of the wavelet coefficients of the layer $\mathrm{D}_{1}$ and layer $\mathrm{D}_{2}$ of the selected voltage and current as well as their corresponding differences, were adopted to constitute the characteristic vector space of neural network. The specific processing of characteristic parameters by this islanding detection method is thus described here: Provide two-scale wavelet decomposition for the collected inverter output voltage and inverter output current signals respectively, extract the wavelet coefficients of the high-frequency components of the two scales and solve their absolute values; respectively calculate the mean values of the wavelet coefficients within a voltage period; denote the absolute mean values of voltage signal wavelet coefficients as $\mathrm{D}_{\mathrm{u} 1}$ and $\mathrm{D}_{\mathrm{u} 2}$, denote the absolute mean values of current signal wavelet coefficients as $\mathrm{D}_{\mathrm{i} 1}$ and $\mathrm{D}_{\mathrm{i} 2}$, and use the six signals, $\mathrm{D}_{\mathrm{u} 1}, \mathrm{D}_{\mathrm{u} 2}, \mathrm{D}_{\mathrm{i} 1}$ and $\mathrm{D}_{\mathrm{i} 2}$ as well as their corresponding differences $\left(\mathrm{D}_{\mathrm{u} 1}-\mathrm{D}_{\mathrm{i} 1}\right)$ and $\left(D_{\mathrm{u} 2}-\mathrm{D}_{\mathrm{i} 2}\right)$, to constitute the characteristic vector space of neural network [12].

\subsection{Structural DESIGN AND ALGORITHM IMPROVEMENT OF NEURAL NETWORK}

After lifting wavelet transform and processing, the values of the characteristic parameters will change significantly under different loading conditions, so it is very difficult to judge the islanding state and non-islanding state of the DG system through setting thresholds; therefore, a powerful system identification tool should be introduced in such case. By virtue of their unique learning ability and approximation function, artificial neural networks can be used to identify any non-linear system. Among them, BP neural network has received the closest attention and been most widely applied, so BP neural network was adopted by this paper for the mode identification of the working state of the DG system. BP neural network is a multi-layer feed-forward network trained by the error back-propagation algorithm, and the topological structure of its network model consists of input layer, hidden layer and output layer.

The number of input layer nodes of BP network is generally equal to the number of vector dimensions of the sample to be trained, and the number of output layer nodes adopts the number of categories in the classification network. There were six signals in the characteristic vector space of neural network selected by this paper, so the number of input layer nodes was set at 6 . Given that output only classifies the current working state of the DG system, i.e., islanding state and non-islanding state, the number of output layer nodes was set at 1 [13].

Setting the number of hidden layer nodes is the key, because if the number of nodes is insufficient, there will be a low fault tolerance and a low ability of identifying unlearned samples, and, if the number of nodes is excessive, the duration of network training will be prolonged. Usually, the number of hidden layer nodes can be designed with reference to the following formula:

$$
l=\sqrt{n+m}+a
$$

Where 1 is the number of hidden layer nodes, $n$ is the number of input nodes, $\mathrm{m}$ is the number of output nodes, and $\alpha$ is a constant between 1 and 10. According to multiple trainings and experiments, when the number of hidden layer nodes is set at 12 , there will be relatively satisfactory training speed and identification effects, so the number of hidden layer nodes was set at 12 .

To reduce signal collection quantity and improve detection accuracy, the neural network structure adopts the method of redundant design, that is, two identical neural network modules are designed for islanding detection, so that anti-islanding protection will be provided only when the islanding state has been detected by both modules in succession; otherwise, no control signal for anti-islanding protection will be produced. In this way, in the case of short circuit, voltage reduction or other sudden changes having occurred at a non-zero crossing point of voltage in the grid, the transient strong disturbance signal hereby generated can be prevented from causing the neural network to make a misjudgment. Meanwhile, it is not necessary in this case to massively collect the voltage and current samples at different moments of mutations for the purpose of avoiding misjudgments, which as a result reduces the collection quantity of signals and lightens the calculation burden.

The most common learning algorithm of BP neural network is the steepest descent static optimization algorithm with a momentum term, and its weight adjustment formula is:

$$
\Delta W(n)=\eta \frac{\partial E}{\partial W}(n)+\alpha \Delta W(n-1)
$$

Where $\Delta W(n)$ is the adjustment amount of this iterative weight; $\alpha \Delta W(n-1)$ is the momentum term, where $\alpha$ is the momentum factor $(0<\alpha<1), E$ is the learning error, and $\eta$ is the learning rate (also referred to as step size; here it is a fixed value) [14]. The disadvantages of this algorithm mainly include:

(1) Seen from the error curved surface, in a flat zone, an excessively small $\eta$ will increase the number of trainings; in a zone with intensive error changes, an excessively large $\eta$ will result in vibrations and increase the number of iterations. Thus, adopting a fixed value for $\eta$ makes it extremely difficult to ascertain an optimal learning rate that is always suitable.

(2) Given that the activation function adopted by BP network is sigmoid, when a nerve cell output approaches the saturation zone of $\mathrm{S}$ function, the gradient becomes very small, causing the adjustment amounts of related weights to 
be almost zero and making it extremely difficult to get rid of the local minimum state, as a result of which the network learning rate becomes poor or even unable to converge.

With regard to the above disadvantages, this paper adopts a dynamic variable-step size learning algorithm. In addition, reducing the sensitivity of neural network can not only improve the performance of neural network but also increase its learning efficiency, and the sensitivity of neural network is related to its weight adjustment. On that account, for the purpose of algorithm modification, the author adopts the algorithm that combines self-adaptive variable step size and reduced neural network sensitivity. See the modified algorithm below:

$$
\eta=k_{1} \eta_{0}+k_{2}\left[2 O_{k}(n)-1\right]^{2}+k_{3} \operatorname{sign}\left[\frac{\partial E}{\partial W}(n) \cdot \frac{\partial E}{\partial W}(n-1)\right]
$$

It can be seen from the above formula that the learning rate $\eta$ consists of three stacked parts.

In the first part $k_{1} \eta_{0}, \eta_{0}$ is a fixed component and is set at $0.3 ; k_{1}$ varies with the size of the training error $E$. When the absolute value of the training error $|\mathrm{E}|>15 \%, k_{1}$ is set at 2 to increase the weight adjustment amount, thus to rapidly change neural network output to reduce the error; when $5 \%<|\mathrm{E}|<15 \%, k_{1}$ is set at 1.5 to reduce neural network sensitivity and prevent network output overshoot and backward error; when $|\mathrm{E}|<5 \%, k_{1}$ is set at 1 to reduce network sensitivity again and quickly reduce the training error to the allowed value.

In the second part of $(10), O_{k}(n)$ represents the actual output value of the neural network node of this iteration, and, in sigmoid function, it ranges between 0 and 1 . Thus, when the nerve cell output approaches the saturation zone of $\mathrm{S}$ function, $O_{k}(n)$ is approximate to 0 or 1 , while $\left[2 O_{k}(n)-1\right] 2$ is approximate to the maximum value 1 ; the farther the nerve cell output away from the saturation zone, the lower the $\left[2 O_{k}(n)-1\right] 2$ value. Thus, the second component of step size calculation dynamically changes with nerve cell output, and, when approaching the saturation zone of $S$ function, correspondingly increasing the step size helps to increase the gradient change rate and accelerates the training. It is proper to set $k 2$ between 0.05 and 0.15 .

The third part of (10) dynamically increases or decreases the learning rate $\eta$ according to the symbol of the gradient direction $\partial E / \partial W(n)$ of two iterations, and $k_{3}$ is set at 0.1 after multiple experiments. Increasing this component in $\eta$ aims to eliminate the complex variable canyon area on the error curved surface; fixed step size, due to its inability to adapt to complex variations, will result in the reduction of convergence speed. The basic idea is that, under the trend of gradient reduction, when the gradient direction symbols of two continuous iterations are opposite, it suggests the excessively quick gradient reduction, so the step size should be reduced, that is, subtracting 0.1 from $\eta$; contrarily, when the gradient direction symbols are the same, it suggests that gradient reduction can be further accelerated, in which case the step size should be increased by 0.1 .

The authors continuously tested a group of samples for ten times by the traditional learning algorithm, and the mean number of iterations was 7,432 . When the method proposed by the author was adopted to test the same samples, the mean number of iterations declined to 2,128. It is thus clear that the modified algorithm remarkably reduces the number of iterations of neural network training, and further significantly shortens the training time.

\subsection{Simulation modelling and analysis of simulation results}

The simulation uses a single phase grid-connected DG system, and the MATLAB model is shown in Fig.2. In this figure, L1 is the local RLC parallel load, and the grid voltage is supplied by the A-phase of the three-phase programmable power supply, which can realize the voltage mutation or join the harmonics. The PI PWM module is a PI control and a PWM pulse generating circuit, the grid-connected inverter adopts current control, and the specified value of current amplitude is 20A. The wave_nn1 module is the islanding detection module which is based on lifting wavelet and neural network, the internal structure of this module is shown in Fig.1. The structure of islanding detection part in wave_nn2 module is the same as that of wave_nn1 module, which is used for the secondary judgment on the system state. When the wave_nn1 module hasn't detected the islanding state, the neural network in wave_nn2 is not put into work, the value of output signal out1 of wave_nn2 module is equal to that of output signal out of module wave_nn1, it is a high level; If wave_nn1 module detects a system islanding state, it will start the neural network in wave_nn2 to make the secondary judgment of the system state. If the result of secondary judgment is still an islanding state, the output signal outl of wave_nn2 module goes low, then the trigger pulse of the inverter is blocked, and the islanding protection of the system is implemented; if the result of secondary judgment is not an islanding state, the output signal out 1 remains high level, and does not implement the islanding protection.

In Fig.2, five different states has been designed to test the effect of islanding detection: (1) islanding state, the breaker S1 in Fig.2 is disconnected and the DG system is separated from the main grid to form an islanding; (2) load mutation status, the switch S2 in this figure is suddenly closed from the disconnected state, and the load L2 is added into the circuit; (3) harmonic disturbance state, 4\% pu 3rd harmonic and $2 \% \mathrm{Pu} 5$ th harmonic is added to the grid voltage to implement the disturbance of the circuit; (4) short-circuit state, the switch S3 in Fig.2 is closed from the disconnected state, so that the output of the inverter is short to ground; (5) grid voltage mutation state, the grid voltage suddenly dropped to $60 \%$ of the original. 


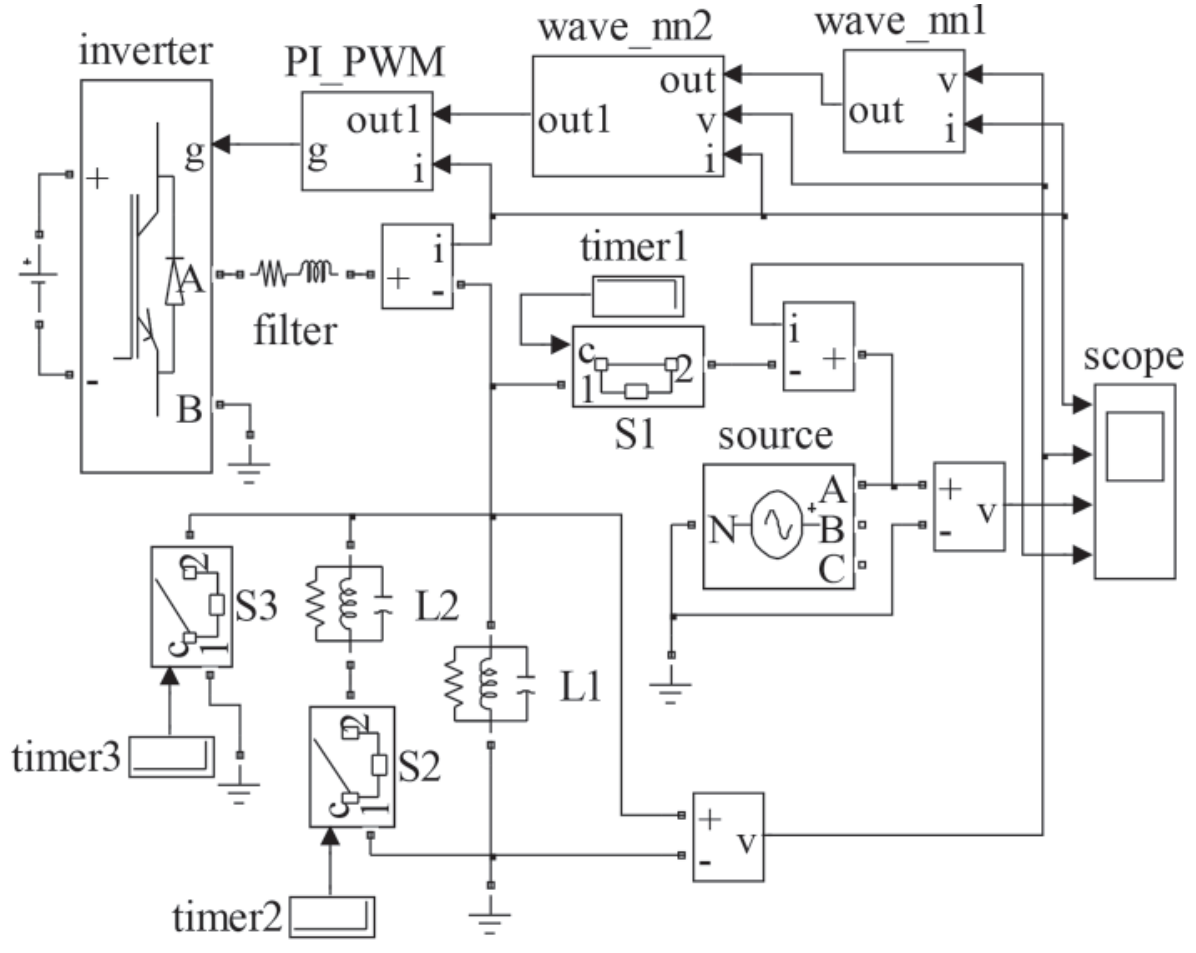

Fig.2: Simulation model of islanding detection

block the trigger pulse of the inverter, so the output current and output voltage of the inverter are reduced to 0 , which means that the islanding state has been successfully detected, and the detecting time is 0.04 seconds, which meets the detecting time requirement of national standard $\mathrm{GB} / \mathrm{T}$ 19939-2005 [15].

Fig.3(b) is the waveform when the local load has sudden change. The figure shows that after 0.04 seconds the grid current becomes smaller due to local load shunt increases. Because it is a non-islanding state, the islanding protection has not been provided, so the output voltage and output current of the inverter remain unchanged.

Fig.3(c) is the waveform when has the harmonic disturbance. The harmonic component is added to the grid voltage at the moment of 0.04 seconds, so after 0.04 seconds the grid current has a certain distortion. Again, because it is a non-islanding state, the

After the simulation model is established, the BP network can be trained, and the training process is as follows: (1) The islanding state is set to mode 1 , the non-islanding states such as short-circuit, load mutation, voltage mutation and harmonic disturbance are all set to mode 2; (2) Set the local RLC parallel load for a wide variety of parameter values, and in each load parameters condition, 7 groups inverter output voltage and inverter output current signals in islanding state and each non-islanding states are collected respectively, the samples of input characteristic vector of neural network is obtained after the wavelet transform and data processing, 5 groups of characteristic vectors are used as training samples of BP neural network, and 2 groups of characteristic vectors are used as test sample of BP network; (3) Neural network is trained with the training sample, and the training effect of neural network is tested by the test sample. When the test results show that the neural network can accurately identify the islanding and non-islanding mode and then end the training. Afterwards, we can use the trained neural network to carry on the islanding detection simulation test. The test parameter of local load $\mathrm{L} 1$ is set to $\mathrm{P}_{\mathrm{L}}=1500 \mathrm{~W}$, $\mathrm{Q}_{\mathrm{L}}=\mathrm{Q}_{\mathrm{C}}=1500 \mathrm{Var}$, which has not been trained by the neural network beforehand. The MATLAB simulation waveforms are shown in Fig.3.

Fig.3(a) is the waveform when the breaker S1 is disconnected at the moment of 0.04 seconds. Due to the formation of an islanding state, the grid current is reduced to 0 at 0.04 seconds, the output signal of wave_nn2 module of Fig. 2 changes from high level to low level at 0.08 seconds to
DG system does not carry out islanding protection, so the inverter output current remains unchanged.

Fig.3(d) is the waveform when a short-circuit occurs. At 0.04 seconds the grid current becomes very large because of short-circuit, and the inverter output voltage is approximately zero, due to the non-islanding state, islanding protection does not carry out, so the inverter output current remains unchanged.

Fig.3(e) is the waveform when has grid voltage mutation. A non-zero-crossing moment of grid voltage (here set to 0.041 seconds) is selected when the grid voltage dip $40 \%$, so the grid voltage and the inverter output voltage have significant change in 0.041 seconds, also since the non-islanding state and without islanding protection, the inverter output current remains unchanged.

In order to prove the necessity of redundancy design of neural network module, the wave_nn2 module in Fig. 2 is removed, the same non-zero-crossing moment of grid voltage (that is 0.041 seconds) is selected to make the grid voltage dip $40 \%$, Fig. 4 is the simulation waveform. The figure shows that the inverter output current is reduced to zero due to the islanding protection malfunction. To compare Fig.4 and Fig.3(e), because the grid voltage dips, the grid current forms a large impact current in 0.041 seconds, so that at this moment the extracted values of characteristic parameters of grid voltage sag state are similar to that of islanding state, and the neural network is likely to mistakenly judge the voltage mutation as a islanding occurrence. Through several tests, authors found 

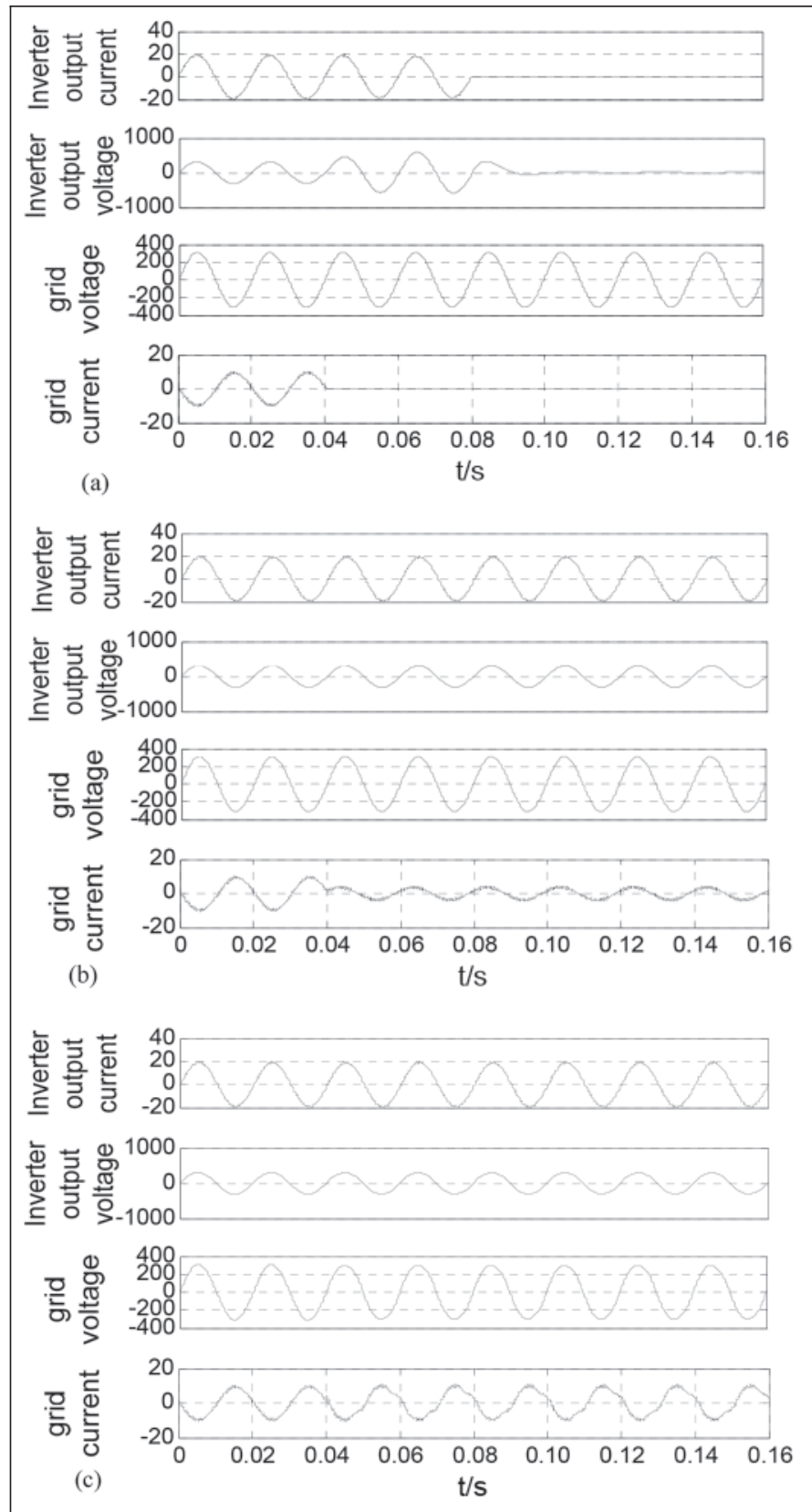

that for any voltage mutational event occurred in non-zerocrossing moment, as long as the sample has not been learned, the neural network has the possibility of misjudgment. It is similar to the voltage mutation, the short-circuit event occurred in non-zero-crossing moment may also be misjudged by neural network. Using secondary judgment in the next voltage cycle, the impact of the mutation signal has passed, no longer affect the work of the neural network, thereby improving the accuracy of pattern recognition of neural network. At the same time, it can reduce the collected amount of training sample, and save the training time of neural network.

The islanding detection method proposed in this paper does not add perturbation to signals, and overcomes the disadvantages of the active islanding detection method which
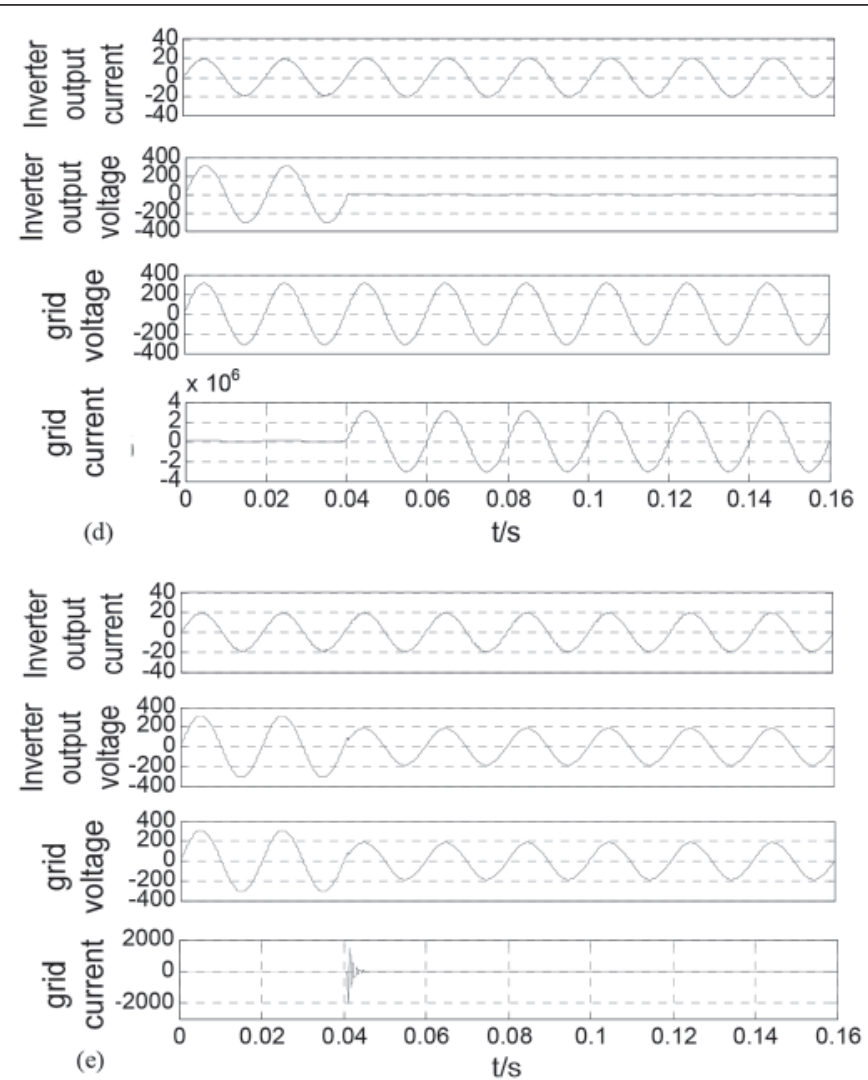

Fig.3: Simulation waveform of islanding detection

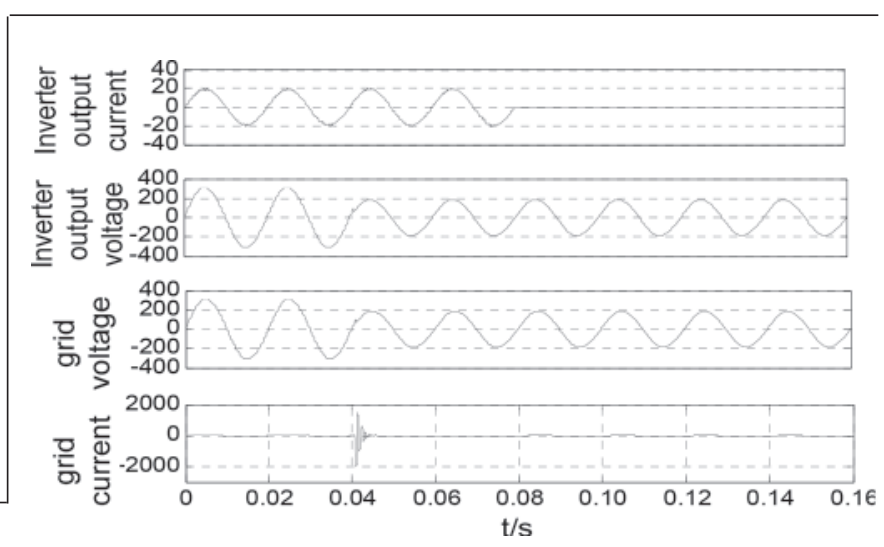

Fig.4 contrast waveform for grid voltage mutation

can affect the power quality. Changing the parameters of local load L1 many times to carry out simulation test under the load matching and load mismatch conditions, all can effectively detect the occurrence of islanding. Therefore, compared to the traditional passive islanding detection method, the proposed method has the advantages of fast detection speed and small NDZ. Moreover, the reliability of detection is improved by using secondary judgment of islanding.

\subsection{Analysis of experimental results}

According to the simulation model, a set of experimental apparatus was designed. In order to verify the detection 


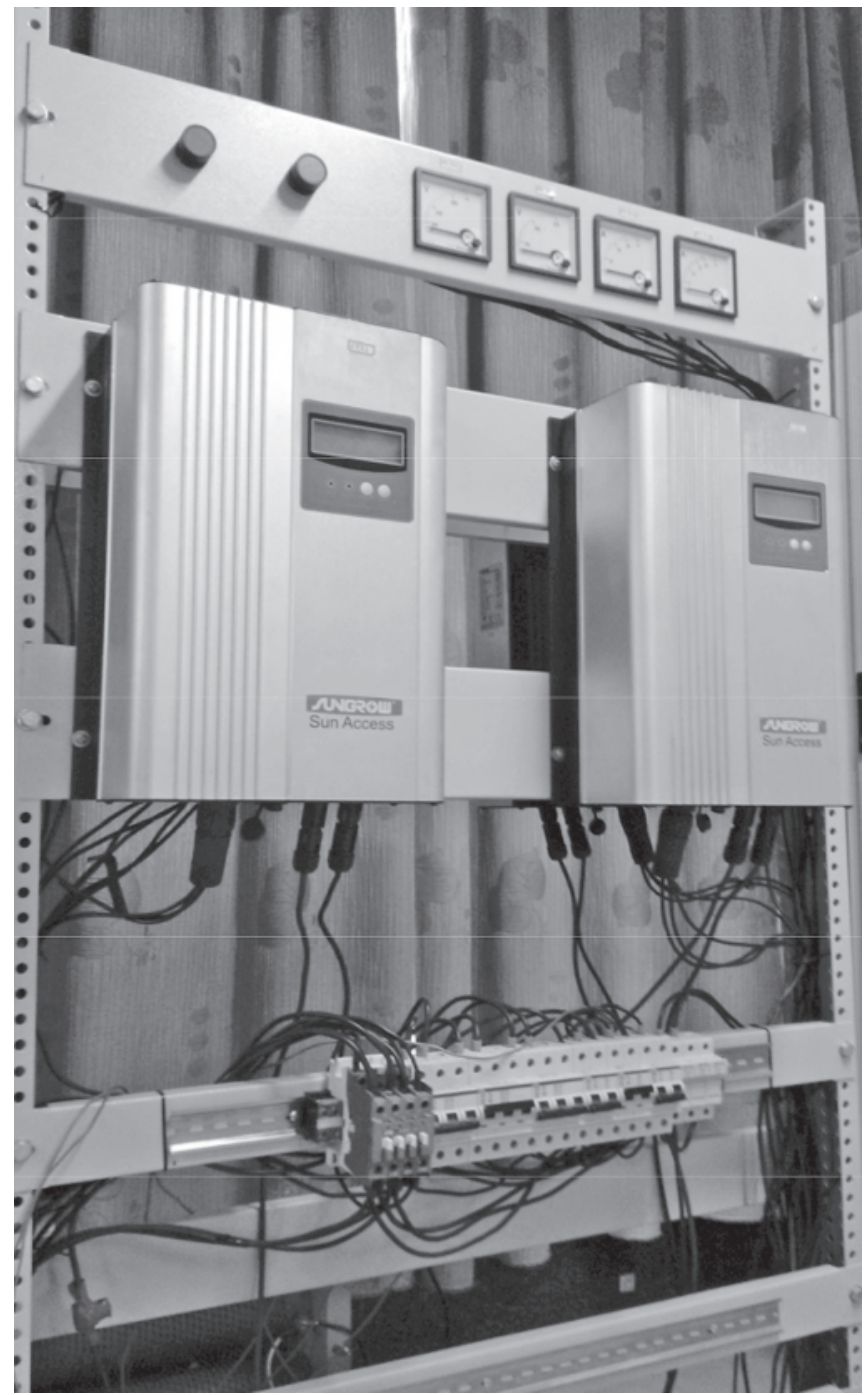

Fig.5: Experimental apparatus of islanding detection

performance of the proposed islanding detection method under the condition of multi grid-connected inverters, 3 SG1K5TL photovoltaic inverters are used in the experimental apparatus, as shown in Fig.5. In DG system, the signal is mainly interfered by low order harmonics such as the 3rd and 5th harmonic, and the wavelet decomposition in this paper only have two scales, that is the high-frequency components of a signal is extracted to identify DG system state, along with the use of secondary judgment, so the neural network never has misjudgment of system state in the harmonic disturbance experiment. Therefore, the analysis is focused on the experimental results of the island state, the load mutation status and the grid voltage mutation state. The experimental parameters are set as follows: the output power of the 3 inverters are all $1000 \mathrm{~W}$, the local load is set to $\mathrm{P}_{\mathrm{L}}=3000 \mathrm{~W}$, $\mathrm{Q}_{\mathrm{L}}=\mathrm{Q}_{\mathrm{C}}=3000 \mathrm{var}$, the inverter output active power is close to matching the active power consumed by local load.

Fig.6 is the experimental waveform when the islanding occurs. In this figure, waveform 1 is the grid current, and

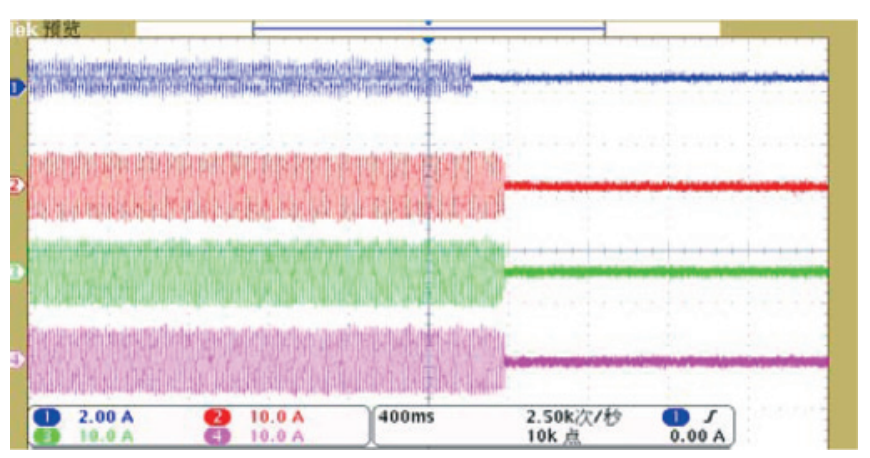

Fig.6: Waveform for islanding occurred in system

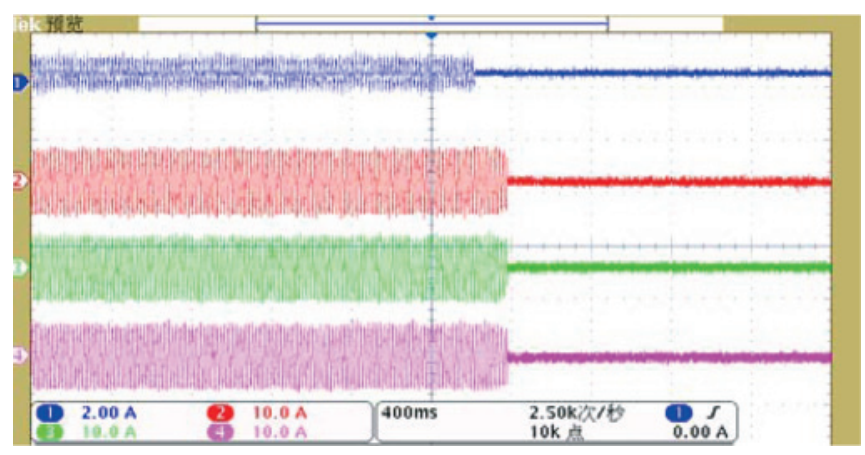

Fig.7: Waveform for local loads mutation

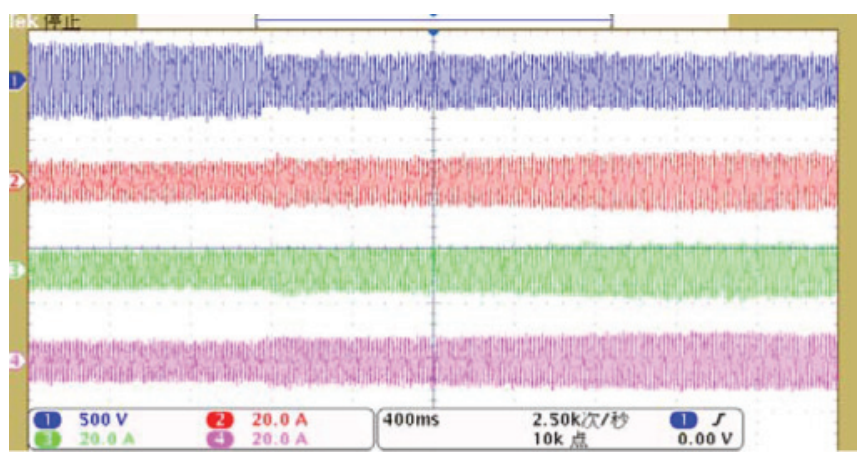

Fig.8: Waveform for voltage grid mutation

waveform 2, 4, 3 are the output currents of the 3 inverters. The figure shows that after the grid current is reduced to zero, that is after the islanding is occurred, the inverter output currents immediately dropped to 0 , which means the inverters have successfully detected the islanding and have implemented protective measures.

Fig.7 is the experimental waveform when the local load has sudden change. In this figure, the four kinds of waveforms are the same as those in Fig.6, the local load suddenly increases $1000 \mathrm{~W}$ during the experiment. As can be seen from the figure, the grid current increases suddenly when the load mutates, because it is a non-islanding state, the islanding protection does not carry out, and the output currents of the inverters remains unchanged.

Fig. 8 is the experimental waveform of grid voltage plunged by $30 \%$. In this figure, waveform 1 is the grid 
voltage, and waveforms 2, 4, 3 are the output currents of the 3 inverters. The figure shows, after a sudden drop of grid voltage, the inverter output currents do not drop to zero as in Fig.6, indicating that the neural network correctly identifies the non-islanding state there is no islanding protection error.

The above experimental results show that the proposed method can detect the islanding quickly and accurately, and the disturbance signals such as load mutation and grid voltage mutation will not cause disoperation. In addition, this method can overcome the shortcoming of traditional passive detection method which will have NDZ under the condition of load matching.

\subsection{Conclusions}

This paper proposes a novel passive islanding detection method based on lifting wavelet and neural network. This method first extracts characteristic parameters from related signals of the DG system through lifting wavelet transform, and then conducts the pattern recognition of these extracted characteristic parameters via BP neural network, so as to judge if the DG system is at the islanding state. As indicated by the simulation and experiment results, the islanding detection method proposed by this paper, by virtue of the excellent performance of lifting wavelet in extracting the transient characteristics of the power system and the powerful mode identification ability of neural network, can accurately judge and identify the islanding state and various non-islanding states. As indicated by the simulation and experiment results under multiple loading conditions of matching and mismatching between the grid-connected inverter and the local load power, this method is featured by high detection speed and small NDZ. In addition, given that the proposed islanding detection method does not introduce any disturbance variable into the control signal, it does not adversely affect electric energy quality.

\section{Conflict of interest}

This article content has no conflict of interest.

\section{Acknowledgement}

This work is supported by the natural science foundation project of Anhui Province (160805ME120), the University Natural Science Research project of Anhui Province (KJ2015A245), and research fund of Tongling University (2014tlxyrc03)

\subsection{References}

[1] Serban E., Pondiche C., Ordonez M.,(2015): “Islanding detection search sequence for distributed power generators under AC grid faults", IEEE Transactions on Power Electronics, vol. 30, pp.3106-3121, June.
[2] Dong Dong, Bo Wen, Mattavelli P., Boroyevich D., Yaosuo Xue, (2014): "Modeling and design of islanding detection using Phase-Locked Loops in three-phase grid-interface power converters", IEEE Journal of Emerging and Selected Topics in Power Electronics, vol. 2, pp. 1032-1040, April.

[3] Guo Y., Li K., Laverty D.M., Xue Y. (2015): "Synchrophasor-based islanding detection for distributed generation systems using systematic principal component analysis approaches", IEEE Transactions on Power Delivery, vol. 30, pp.2544-2552, June.

[4] Al Hosani M., Zhihua Qu, Zeineldin H.H., (2015): "Development of dynamic estimators for islanding detectionof inverter-based DG", IEEE Transactions on Power Delivery, vol. 30, pp. 428-436, January.

[5] Xiaolong Chen, Yongli Li. (2014): “An islanding detection algorithm for inverter-based distributed generation based on reactive power control", IEEE Transactions on Power Electronics, vol. 29, pp. 46724683, September.

[6] Samet H., Hashemi F., Ghanbari T. (2015):“'Islanding detection method for inverter-based distributed generation with negligible non-detection zone using energy of rate of change of voltage phase angle", IET Generation, Transmission \& Distribution, vol.9, pp. 2337-2350, November.

[7] Alam M.R., Muttaqi K.M., Bouzerdoum A. (2014): “An approach for assessing the effectiveness of multiplefeature-based SVM method for islanding detection of distributed generation", IEEE Transactions on Industry Applications, vol. 50, pp. 2844-2852, April.

[8] Jun Zhang, Dehong Xu, Guoqiao Shen, Ye Zhu, Ning He, Jie Ma(2013):“An improved islanding detection method for a grid-connected inverter with intermittent bilateral reactive power variation", IEEE Transactions on Power Electronics, vol. 28, pp. 268- 278, January.

[9] Lidula, A. D. Rajapakse (209): "Fast and reliable detection of power islands using transient signals" Fourth International Conference on Industrial and Information Systems, pp.1-6.

[10] Samui A., Samantaray S.R. (2013): "Wavelet singular entropy-based islanding detection in distributed generation", IEEE Transactions on Power Delivery, Vol.28, pp.411-418, January.

[11] Lidula N.W.A., Rajapakse A.D. (2012): “A patternrecognition approach for detecting power islands using transient signals-part II: performance evaluation", IEEE Transactions on Power Delivery, vol.27, pp.1071-1080, March. 
[12] Yang Tao, Feng Yongxin, Ren Yong, Tang Lei, Li Yanghai(2012): "Parameter Identification of Steam Turbine Speed Governor System”, 2012 Asia-Pacific Power and Energy Engineering Conference, pp. 1-8.

[13] Kuei-Hsiang Chao, Min-Sen Yang, Chin-Pao Hung, (2013): "Applying a CMAC neural network to a photovoltaic system islanding detection", International Conference on Machine Learning and Cybernetics, Tianjin, pp. 259-264.
[14] Wenhao Huang, Haikun Hong, Guojie Song, Kunqing Xie, (2014): "Deep process neural network for temporal deep learning", [14] 2014 International Joint Conference on Neural Networks, pp. 465-472.

[15] S. Wang, H. Yang, L. Wang. (2005): GB/T 19939-2005 technical requirements of grid-connected photovoltaic system, national standard of the people's Republic of China, Nov.

\section{ANALYSIS OF THE EARNINGS MANAGEMENT METHODS OF CHINA'S ELECTRIC POWER COMPANIES BASED ON MODIFIED JONES MODEL}

(Continued from page 90)

[11] Patricia M. Dechow and Douglas J. Skinner,(2000): "Earnings Management: Reconciling the Views of Accounting Academics, Practitioners, and Regulators", Accounting Horizons, 2000, Vol.14 (2), p.235-260.

[12] Katherine A. Guuny, (2010): "The Relation between Earnings Management Using Real Activities Manipulation and Future Performance: Evidence from Meeting Earnings Benchmarks", Contemporary Accounting Research, Vol.27 (3), p.855-888.

[13] Jianqiao Lu(1999): "Empirical Study on the Earnings Management of China's Deficit Listed Companies", Accounting Research, (9), p.25-35.
[14] Zengquan Li and Wenbin Lu, (2003): "The Stability of Accounting Earnings: Discovery and Enlightenment", Accounting Research, (2), p.19-27.

[15] Mian Qin and Wei Zhou,(2003): "The Feature Analysis of Eight Accruals", Capital Markets Magazine, (9), p.14-17+7.

[16] P.M. Dechow, A.P. Sweeney, and R. G. Sloan, (1995): "Detecting Earnings Management", The Accounting Review, Vol.70 (2), p193-225.

[17] Qiulin Quan, (2017): "Intervention Research of New Accounting Standards and Corporate Earnings Management", Finance and Taxation Research, (13), p.182-183.

\section{JOURNAL OF MINES, METALS \& FUELS}

\section{Annual Subscription : \\ Rs.3000 (India); $£ 280.00$ or $\$ 400.00$ (Foreign)}

The Editor

Journal of Mines, Metals \& Fuels

Moon Plaza

2A, 2nd Floor, 62 Lenin Sarani, Taltala,

Kolkata 700013 India

E-mail: bnjournals@gmail.com / pradipchanda@yahoo.co.uk•www.jmmf.info 